\title{
Convergent Properties of Estimators Concerned with a Class of Vague Random Phenomena*
}

\author{
Tokuo FUKUDA $^{\dagger}$
}

In this paper, the author investigates the convergent properties of estimators concerned with expectations for a class of vague random phenomena, where the fuzzy random set is considered as a model of the capricious vague perception of a crisp phenomenon or a crisp random phenomenon.

First, fuzzy random sets as vague random perceptions of non-random or random phenomena are investigated, and their expectations are also considered. Secondly applying the standard Strong Law of Large Numbers(SLLN) for the random elements in a separable Banach space, the convergent properties of estimators for expectations of the proposed fuzzy random sets are examined, and finally they are confirmed numerically by simulation studies.

\section{Introduction}

Fuzzy random sets or fuzzy random variables have been intensively investigated for a long time by many researchers with various definitions motivated by the importance for treating the data exhibiting both vagueness and randomness. For instance, the concept of fuzzy random variables obtained as vague linguistic observations of crisp random data was firstly presented by Kwakernaak[1], and investigated by e.g., Kruse[2]. On the other hand, Puri and Ralescu[3] defined firstly fuzzy random variables as the generalized random sets and discussed their statistical properties by many researchers, e.g., $[4,5]$.

The purpose of this paper is to investigate the convergent properties of estimators concerned with a class of fuzzy random sets, which is considered as a model of the capricious vague perception of a crisp non-random or random phenomenon.

Considering that the vague perception of a crisp phenomenon fluctuates slightly but randomly due to the state of a capricious person's mind[6,7], a class of fuzzy random sets is firstly introduced in Sec. $\mathbf{2}$ for non-random phenomena and in Sec. 3 for random phenomena. In Section 4, applying the standard Strong Law of Large Numbers(SLLN) for the random elements in a separable Banach space, the convergent

\footnotetext{
* Manuscript Received Date: August 29, 2015

This paper is the revised version of that presented in the 46th ISCIE International Symposium on Stochastic Systems Theory and Its Applications(SSS'14) which was held in Nov., 2014.

$\dagger$ Faculty of Management, Otemon Gakuin University; 21-15 Nishi-Ai, Ibaraki, Osaka 567-8502, JAPAN

Key Words: fuzzy random sets, capricious vague perception, expectation, Strong Law of Large Numbers(SLLN).
}

properties of estimators of expectations for the proposed fuzzy random sets are examined. Finally, the convergent properties of proposed estimators are confirmed numerically by simulation studies in Sec. $\mathbf{5}$.

\section{Vague Perceptions of Crisp Phenomena}

First, we consider that the vague perception of a crisp phenomenon fluctuates slightly but randomly due to the state of a capricious person's mind. Hence, the fuzzy set obtained as a vague perception of a crisp phenomenon may be some kind of 'fuzzy random set'. Using the class of fuzzy sets $\mathbb{F}_{\mathrm{cc}}^{(p)}\left(\mathbb{R}^{n}\right)$, the elementary fuzzy random set is described as follows[8]:

[Definition 1] Let $\left(\Omega, \mathcal{A}, P_{u_{\mathrm{o}}}\right)$ be an elementary probability space, where $\Omega=\left\{\omega_{1}, \omega_{2}, \cdots, \omega_{M}\right\} ; \mathcal{A}$ be a $\sigma$-algebra given by the subsets of $\Omega$; and $P_{u_{\mathrm{o}}}$ is a probability measure such that $P_{u_{\mathrm{o}}}\left(\omega_{i}\right)>0$ for each $i=1,2, \cdots, M$. Then, an elementary fuzzy random set as a vague perception of the original point $u_{\mathrm{o}} \in \mathbb{R}^{n}$ is defined by

$$
\widetilde{U}\left(u_{\mathrm{o}}, \omega\right)=\left(\mathbb{R}^{n},\left[\widetilde{U}\left(u_{\mathrm{o}}, \omega\right)\right], s_{\widetilde{U}\left(u_{\mathrm{o}}, \omega\right)}\right) \in \mathbb{F}_{\mathrm{cc}}^{(p)}\left(\mathbb{R}^{n}\right)
$$

with

$$
\left[\widetilde{U}\left(u_{\mathrm{o}}, \omega\right)\right]=\left\{\left[\widetilde{U}\left(u_{\mathrm{o}}, \omega\right)\right]_{\alpha} \mid \alpha \in \mathbb{I}\right\},
$$

where

$$
\begin{array}{r}
s_{\widetilde{U}\left(u_{\mathrm{o}}, \omega\right)}(u)=\left\{u \text { in } \widetilde{U}\left(u_{\mathrm{o}}, \omega\right)\right. \text { coincides with } \\
\text { the original point } \left.u_{\mathrm{o}}\right\} .
\end{array}
$$

Then, we can rewrite eq. (1) by 


$$
\widetilde{U}\left(u_{\mathrm{o}}, \omega\right)=\sum_{i=1}^{M} \mathbf{1}_{\omega_{i}}(\omega) \cdot \widetilde{U}_{i}\left(u_{\mathrm{o}}\right)
$$

where $\mathbf{1}_{\omega_{i}}(\omega)$ is the characteristic function of $\omega_{i}$ given by

$$
\mathbf{1}_{\omega_{i}}(\omega)= \begin{cases}1 & \text { if } \omega=\omega_{i} \\ 0 & \text { otherwise }\end{cases}
$$

and $\widetilde{U}_{i}\left(u_{\mathrm{o}}\right)$ is the fuzzy set given by the triple

$$
\widetilde{U}_{i}\left(u_{\mathrm{o}}\right)=\left(\mathbb{R}^{n},\left[\widetilde{U}_{i}\left(u_{\mathrm{o}}\right)\right], s_{\widetilde{U}_{i}\left(u_{\mathrm{o}}\right)}\right) \in \mathbb{F}_{\mathrm{cc}}^{(p)}\left(\mathbb{R}^{n}\right) .
$$

Hence, we have the relation between $\left[\widetilde{U}\left(u_{\mathrm{o}}, \omega\right)\right]_{\alpha}$ and $\left[\widetilde{U}_{i}\left(u_{\mathrm{o}}\right)\right]_{\alpha}(i=1,2, \cdots, M)$ such that

$$
\left[\widetilde{U}\left(u_{\mathrm{o}}, \omega\right)\right]_{\alpha}=\sum_{i=1}^{M} \mathbf{1}_{\omega_{i}}(\omega) \cdot\left[\widetilde{U}_{i}\left(u_{\mathrm{o}}\right)\right]_{\alpha}
$$

for each $\alpha \in \mathbb{I}$. The measurability of $\widetilde{U}\left(u_{\mathrm{o}}, \omega\right)$ is given through its $\mathcal{A}-\mathcal{B}\left(u_{\mathrm{o}}\right)$ measurability, i.e.,

$$
\widetilde{U}^{-1}\left(u_{\mathrm{o}}, \cdot\right)(\mathrm{B}) \in \mathcal{A} \quad \text { for } \forall \mathrm{B} \in \mathcal{B}\left(u_{\mathrm{o}}\right),
$$

where $\mathcal{B}\left(u_{\mathrm{o}}\right)$ is a $\sigma$-algebra generated by the subsets of $\widetilde{\mathbb{U}}\left(u_{\mathrm{o}}\right)=\left\{\widetilde{U}_{1}\left(u_{\mathrm{o}}\right), \widetilde{U}_{2}\left(u_{\mathrm{o}}\right), \cdots, \widetilde{U}_{M}\left(u_{\mathrm{o}}\right)\right\}$.

Since $\widetilde{U}\left(u_{\mathrm{o}}, \omega\right)$ is a some kind of the random quantity, it should be possible to consider its statistical moments such as its expectation, its variance and so on. Let restrict hereafter the admissible class $\mathfrak{A}$ of the possible random original points to integrable $\mathcal{A}$ measurable ones given as follows:

$$
\begin{aligned}
\mathfrak{A}=\left\{u \mid u(\omega)=\sum_{i=1}^{M} \mathbf{1}_{\omega_{i}}(\omega) \cdot u_{i}\right. \\
\left.\quad \text { and } u_{i} \in \mathbb{R}^{n} \text { for each } i=1,2, \cdots, M\right\} .
\end{aligned}
$$

[Definition 2] Let $\widetilde{U}\left(u_{\mathrm{o}}, \omega\right) \in \mathbb{F}_{\mathrm{cc}}^{(p)}\left(\mathbb{R}^{n}\right)$ be an elementary fuzzy random set. Then, the expectation of $\widetilde{U}$ is given by

$$
\mathcal{E}_{u_{\mathrm{o}}}[\widetilde{U}]=\sum_{i=1}^{M} \widetilde{U}_{i}\left(u_{\mathrm{o}}\right) \cdot P_{u_{\mathrm{o}}}\left(\omega_{i}\right)
$$

with its set representation given by

$$
\left[\mathcal{E}_{u_{\mathrm{o}}}[\widetilde{U}]\right]=\left\{\left[\mathcal{E}_{u_{\mathrm{o}}}[\widetilde{U}]\right]_{\alpha} \mid \alpha \in \mathbb{I}\right\}
$$

and

$$
\begin{aligned}
{\left[\mathcal{E}_{u_{\mathrm{o}}}[\widetilde{U}]\right]_{\alpha} } & =E_{u_{\mathrm{o}}}\left[[\widetilde{U}]_{\alpha}\right] \\
& =\sum_{i=1}^{M}\left[\widetilde{U}_{i}\left(u_{\mathrm{o}}\right)\right]_{\alpha} \cdot P_{u_{\mathrm{o}}}\left(\omega_{i}\right) .
\end{aligned}
$$

The predicate $s_{\mathcal{E}_{u_{\mathrm{o}}}[\widetilde{U}]}$ of $\mathcal{E}_{u_{\mathrm{o}}}[\widetilde{U}]$ is given through

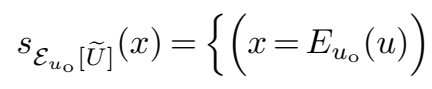

$$
\text { and } \left.\left(u=u_{\mathrm{o}} \text { for some } u \in \mathfrak{A}\right)\right\}
$$

with

$$
E_{u_{\mathrm{o}}}(u)=\sum_{i=1}^{M} u_{i} \cdot P_{u_{\mathrm{o}}}\left(\omega_{i}\right),
$$

where $u=\sum_{i=1}^{M} \mathbf{1}_{\omega_{i}}(\omega) \cdot u_{i}$ is an element of $\mathfrak{A}$, and it should be noted that the probability $P_{u_{\mathrm{o}}}\left(\omega_{i}\right)$ depends on the value of the original point $u_{\mathrm{o}}$.

Then, we have the following proposition (see[6,7]).

[Proposition 1] Let $\widetilde{U}\left(u_{\mathrm{o}}, \omega\right)$ be an n-dimensional elementary random set, i.e., $\widetilde{U} \in \mathbb{F}_{\mathrm{cc}}^{(p)}\left(\mathbb{R}^{n}\right)$. Then, there exists a fuzzy set such that

$$
\mathcal{E}_{u_{\mathrm{o}}}[\widetilde{U}]=\sum_{i=1}^{M} \widetilde{U}_{i}\left(u_{\mathrm{o}}\right) \cdot P_{u_{\mathrm{o}}}\left(\omega_{i}\right) \in \mathbb{F}_{\mathrm{cc}}^{(p)}\left(\mathbb{R}^{n}\right) .
$$

\section{Vague Perceptions of Random Phenomena}

Although there are many crisp non-random phenomena that are perceived vaguely, there are also many crisp phenomena which are themselves randomly changed. In order to consider the vague perceptions of random phenomena, two types of randomness should be considered, one of which is the randomness due to the capricious person's feelings and another of which is the randomness of the phenomena themselves.

The proposed class of fuzzy random sets in Sec. 2 is generalized to that for the vague perceptions of random phenomena, which is the revised version of that given in $[8,9]$. Let $\left(\Omega_{1}, \mathcal{A}_{1}, P_{1}\right)$ be an elementary probability space describing the randomness of capricious persons' minds defined as $(\Omega, \mathcal{A}, P)$ in Definition $\mathbf{1}$, and let $\left(\Omega_{2}, \mathcal{A}_{2}, P_{2}\right)$ be a probability space, on which an original random point $u_{\mathrm{o}} \in \mathbb{R}^{n}$ as the model of a random phenomenon is defined.

Then, the fuzzy random set as a vague perception of the random vector $u_{\mathrm{o}}$ is defined on $(\Omega, \mathcal{A}, P)=$ $\left(\Omega_{1} \times \Omega_{2}, \mathcal{A}_{1} \otimes \mathcal{A}_{2}, P_{1} \times P_{2}\right)$. Let $\widetilde{U}_{M}(\omega) \in \mathbb{F}_{\mathrm{cc}}^{(p)}\left(\mathbb{R}^{n}\right)$ be a fuzzy random set given by

$$
\widetilde{U}_{M}(\omega)=\sum_{i=0, \pm 1, \pm 2, \cdots, \pm M} \mathbf{1}_{\omega_{i}^{(1)}}(\omega) \cdot \widetilde{U}_{i}
$$

where

$$
\mathbf{1}_{\omega_{i}^{(1)}}(\omega)= \begin{cases}1 & \text { if } \omega \in\left\{\omega_{i}^{(1)}\right\} \times \Omega_{2} \\ 0 & \text { otherwise. }\end{cases}
$$

$\left\{\widetilde{U}_{i} ; i=0, \pm 1, \pm 2, \cdots, \pm M\right\}$ in eq. (16) is a collection of fuzzy sets given by

$$
\widetilde{U}_{i}=\left(\mathbb{R}^{n},\left[\widetilde{U}_{i}\right], s_{\widetilde{U}_{i}}\right) \in \mathbb{F}_{\mathrm{cc}}^{(p)}\left(\mathbb{R}^{n}\right)
$$

with

$$
\left[\widetilde{U}_{i}\right]=\left\{\left[\widetilde{U}_{i}\right]_{\alpha} \mid \alpha \in \mathbb{I}\right\}
$$


and $s_{\widetilde{U}_{i}}$ is the predicate associated with the statement such as

$$
\begin{array}{r}
s_{\widetilde{U}_{i}}(u)=\left\{u \in \widetilde{U}_{i}\right. \text { coincides with the original } \\
\text { random point } \left.u_{\mathrm{o}}\right\} .
\end{array}
$$

Assuming that there exists a fuzzy random set $\widetilde{U}(\omega) \in \mathbb{F}_{\mathrm{cc}}^{(p)}\left(\mathbb{R}^{n}\right)$ such that

$$
\lim _{M \rightarrow \infty} \rho_{p}\left(\widetilde{U}(\omega), \widetilde{U}_{M}(\omega)\right)=0 \quad \text { a.s. }
$$

or equivalently

$$
\widetilde{U}(\omega)=\lim _{M \rightarrow \infty} \widetilde{U}_{M}(\omega) \text { a.s. }
$$

the following definition is obtained:

[Definition 3] An extended fuzzy random set $\widetilde{U}(\omega)$ on $(\Omega, \mathcal{A}, P)$ obtained as the capricious vague perception of an original random point $u_{\mathrm{o}}\left(\omega^{(2)}\right)$ on $\left(\Omega_{2}, \mathcal{A}_{2}, P_{2}\right)$ is defined by

$$
\widetilde{U}(\omega)=\left(\mathbb{R}^{n},[\widetilde{U}(\omega)], s_{\widetilde{U}}\right) \in \mathbb{F}_{\mathrm{cc}}^{(p)}\left(\mathbb{R}^{n}\right)
$$

with

$$
[\widetilde{U}(\omega)]=\left\{[\widetilde{U}(\omega)]_{\alpha} \mid \alpha \in \mathbb{I}\right\}
$$

where $s_{\widetilde{U}}$ is the predicate associated with the proposition such as

$$
\begin{aligned}
s_{\widetilde{U}}(u)=\{u \text { in } \widetilde{U} \text { coincides with } \\
\text { the original random point } \left.u_{\mathrm{o}}\right\} .
\end{aligned}
$$

Then, using eq. (16) and eq. (22), we can rewrite $\widetilde{U}(\omega)$ in eq. (23) by

$$
\widetilde{U}(\omega)=\sum_{i=0, \pm 1, \pm 2, \cdots} \mathbf{1}_{\omega_{i}^{(1)}}(\omega) \cdot \widetilde{U}_{i}
$$

where $\left\{\widetilde{U}_{i} ; i=0, \pm 1, \pm 2, \cdots\right\}$ is a collection of fuzzy sets given by eq. (18), and $\mathbf{1}_{\omega_{i}^{(1)}}(\omega)$ is a characteristic function given by eq. (17).

The measurability of $\widetilde{U}$ is given through

$$
\widetilde{U}^{-1}(\mathrm{~B}) \in \mathcal{A}=\mathcal{A}_{1} \otimes \mathcal{A}_{2} \quad \text { for any } \mathrm{B} \in \mathcal{B},
$$

where $\mathcal{B}$ is a $\sigma$-algebra generated by the subsets of $\widetilde{\mathbb{U}}=$ $\left\{\widetilde{U}_{0}, \widetilde{U}_{ \pm 1}, \widetilde{U}_{ \pm 2}, \cdots\right\}$, and the admissible class of possible original random points $\mathfrak{A}_{e}$ is assumed to be given by

$$
\begin{aligned}
\mathfrak{A}_{e}= & \left\{u \mid u(\omega)=\sum_{i=0, \pm 1, \pm 2, \cdots} \mathbf{1}_{\omega_{i}^{(1)}}(\omega) \cdot \xi\left(\omega^{(2)}\right) ;\right. \\
& \left.\xi \text { is the integrable on }\left(\Omega_{2}, \mathcal{A}_{2}, P_{2}\right)\right\} .
\end{aligned}
$$

Applying the similar procedure as that for $\widetilde{U}\left(u_{\mathrm{o}}, \cdot\right)$, we can show that the expectation of a fuzzy random set $\widetilde{U}$ may be given as follows:
[Definition 4] Let $\widetilde{U}=\left(\mathbb{R}^{n},[\widetilde{U}], s_{\widetilde{U}}\right)$ be an extended fuzzy random set given by eq. (23). Then, the expectation of $\widetilde{U}$ is given by

$$
\mathcal{E}[\widetilde{U}]=\left(\mathbb{R}^{n},[\mathcal{E}[\widetilde{U}]], s_{\mathcal{E}[\widetilde{U}]}\right)
$$

with

$$
[\mathcal{E}[\widetilde{U}]]=\left\{E\left[[\widetilde{U}]_{\alpha}\right] \mid \alpha \in I\right\},
$$

where $s_{\mathcal{E}[\widetilde{U}]}$ is the predicate associated with the proposition given by

$$
\begin{aligned}
s_{\mathcal{E}[\widetilde{U}]}(x)=\{x \text { coincides with } \\
\text { the expectation of } \left.u_{\mathrm{o}}\right\},
\end{aligned}
$$

and $[\mathcal{E}[\widetilde{U}]]$ is the set representation of $\mathcal{E}[\widetilde{U}]$ given through

$$
E\left[[\widetilde{U}]_{\alpha}\right]=\sum_{i=0, \pm 1, \pm 2, \cdots}\left[\widetilde{U}_{i}\right]_{\alpha} \cdot P\left(\omega_{i}^{(1)}\right),
$$

where $P\left(\omega_{i}^{(1)}\right)$ is the marginal probability, i.e., $P\left(\omega_{i}^{(1)}\right)$ $=P\left(\left\{\omega_{i}^{(1)}\right\}, \Omega_{2}\right)$.

Let here $\mathcal{S}$ be the sub $\sigma$-algebra of $\mathcal{A}$ consisting all cylinder sets of the form $A=\Omega_{1} \times A^{(2)}$ with $A^{(2)} \in$ $\mathcal{A}_{2}$. Then, the conditional expectation of $\widetilde{U}$ concerned with $\mathcal{S}$ should be given as follows:

$$
\mathcal{E}[\widetilde{U} \mid \mathcal{S}]=\left(\mathbb{R}^{n},[\mathcal{E}[\widetilde{U} \mid \mathcal{S}]], s_{\mathcal{E}[\widetilde{U} \mid \mathcal{S}]}\right)
$$

with

$$
[\mathcal{E}[\widetilde{U} \mid \mathcal{S}]]=\left\{E\left[[\widetilde{U} \mid \mathcal{S}]_{\alpha}\right] \mid \alpha \in I\right\} .
$$

[Proposition 2] Let $\widetilde{U}=\left(\mathbb{R}^{n},[\widetilde{U}], s_{\widetilde{U}}\right)$ be an extended fuzzy random set given by eq. (23). Then, it follows

$$
\mathcal{E}[\widetilde{U}]=\mathcal{E}[\mathcal{E}[\widetilde{U} \mid \mathcal{S}]]
$$

where $\mathcal{E}[\widetilde{U} \mid \mathcal{S}]$ defined by eq. (33) is given by

$$
\mathcal{E}[\widetilde{U} \mid \mathcal{S}]=\sum_{i=0, \pm 1, \pm 2, \cdots} \widetilde{U}_{i} \cdot P\left(\omega_{i}^{(1)} \mid \mathcal{S}\right) .
$$

\section{Convergent Properties of Estimators}

The distribution of an elementary fuzzy random set $\widetilde{U}\left(u_{\mathrm{o}}, \cdot\right) \in \widetilde{\mathbb{U}}\left(u_{\mathrm{o}}\right)$ is a probability measure on $\widetilde{\mathbb{U}}\left(u_{\mathrm{o}}\right)$ defined by

$$
P_{\widetilde{U}\left(u_{\mathrm{o}}, \cdot\right)}(\mathrm{B})=P\left(\widetilde{U}^{-1}\left(u_{\mathrm{o}}, \mathrm{B}\right)\right)
$$

for any $\mathrm{B} \in \mathcal{B}\left(u_{\mathrm{o}}\right)$. Let $\mathcal{A}_{\widetilde{U}\left(u_{\mathrm{o}},{ }^{\prime}\right)}$ be the $\sigma$-algebra generated by $\widetilde{U}^{-1}\left(u_{\mathrm{o}}, \mathrm{B}\right)$, i.e.,

$$
\mathcal{A}_{\widetilde{U}\left(u_{\mathrm{o}}, \cdot\right)}=\sigma\left\{\widetilde{U}^{-1}\left(u_{\mathrm{o}}, \mathrm{B}\right) ; \mathrm{B} \in \mathcal{B}\left(u_{\mathrm{o}}\right)\right\} .
$$

Then, fuzzy random sets $\left\{\widetilde{U}^{i}\left(u_{\mathrm{o}}, \cdot\right) ; i=1,2, \cdots\right\}$ are said to be independent if $\left\{\mathcal{A}_{\widetilde{U}^{i}\left(u_{\mathrm{o}}, \cdot\right)} ; i=1,2, \cdots\right\}$ are independent, and identically distributed(i.i.d.) if all 
$\left\{P_{\widetilde{U}^{i}\left(u_{o}, \cdot\right)} ; i=1,2, \cdots\right\}$ are identical, and independent identically distributed (denoted by i.i.d. simply), if they are independent and identically distributed.

Then, it can be shown that every elementary fuzzy set $\widetilde{U} \in \mathbb{F}_{\mathrm{cc}}^{(p)}\left(\mathbb{R}^{n}\right)$ is embedded into the separable Banach space $\mathbb{L}^{p}\left(\overline{\bar{I}} \times \mathbb{S}^{n-1},\|\cdot\|_{p}\right)$ by the mappings as follows $[8,10]$ :

$$
\begin{aligned}
& j_{\mathbb{F}_{\mathrm{cc}}^{(p)}\left(\mathbb{R}^{n}\right)}: \mathbb{F}_{\mathrm{cc}}^{(p)}\left(\mathbb{R}^{n}\right) \rightarrow \mathbb{L}^{p}\left(\overline{\mathbb{I}} \times \mathbb{S}^{n-1},\|\cdot\|_{p}\right), \\
& j_{\mathbb{F}_{\mathrm{cc}}^{(p)}\left(\mathbb{R}^{n}\right)}(\widetilde{U})=\widetilde{\operatorname{sp}}(\widetilde{U}, \alpha, x)
\end{aligned}
$$

with the norm $\|\cdot\|_{p}$

$$
\left\|j_{\mathbb{F}_{\mathrm{cc}}^{(p)}\left(\mathbb{R}^{n}\right)}(\widetilde{U})-j_{\mathbb{F}_{\mathrm{cc}}^{(p)}\left(\mathbb{R}^{n}\right)}(\widetilde{V})\right\|_{p}=\rho_{p}(\widetilde{U}, \widetilde{V}) .
$$

Then, we can make use of the standard Strong Law of Large Numbers(SLLN) in a separable Banach space[11]. [Proposition 3] Let $(B,\|\cdot\|)$ be a separable Banach space and let $\left\{X^{i} ; i=1,2, \cdots\right\}$ be a sequence of i.i.d. Borel random variable distributed as $X$ with values in $B$. Then,

$$
\left\|\frac{1}{N} \sum_{i=1}^{N} X^{i}-E(X)\right\|_{p} \rightarrow 0 \quad \text { a.s. } \quad \text { as } N \rightarrow \infty
$$

if and only if $E(\|X\|)<+\infty$.

Substituting $\widetilde{\mathrm{sp}}\left(\widetilde{U}^{i}\left(u_{\mathrm{o}}, \cdot\right), \alpha, x\right), \widetilde{\mathrm{sp}}\left(\widetilde{U}\left(u_{\mathrm{o}}, \cdot\right), \alpha, x\right)$ for $X^{i}$ and $X$ respectively, we have

$$
\begin{aligned}
& \frac{1}{N} \sum_{i=1}^{N} \widetilde{\operatorname{sp}}\left(\widetilde{U}^{i}\left(u_{\mathrm{o}}, \cdot\right), \alpha, x\right) \\
& =\widetilde{\operatorname{sp}}\left(\frac{1}{N} \sum_{i=1}^{N} \widetilde{U}^{i}\left(u_{\mathrm{o}}, \cdot\right), \alpha, x\right)
\end{aligned}
$$

and

$$
E\left(\widetilde{\operatorname{sp}}\left(\widetilde{U}\left(u_{\mathrm{o}}, \cdot\right), \alpha, x\right)\right)=\widetilde{\operatorname{sp}}\left(\mathcal{E}_{u_{\mathrm{o}}}\left[\widetilde{U}\left(u_{\mathrm{o}}, \cdot\right)\right], \alpha, x\right) .
$$

Furthermore, we have

$$
\begin{aligned}
& E\left(\left\|\widetilde{U}\left(u_{\mathrm{o}}, \cdot\right)\right\|\right)=E\left(\rho_{p}\left(\widetilde{U}\left(u_{\mathrm{o}}, \cdot\right),\{0\}\right)\right) \\
& \quad=\sum_{i=1}^{M} \rho_{p}\left(\widetilde{U}_{i}\left(u_{\mathrm{o}}\right),\{0\}\right) \cdot P_{u_{\mathrm{o}}}\left(\omega_{i}\right)<+\infty
\end{aligned}
$$

because of $\widetilde{U}_{i}\left(u_{\mathrm{o}}\right) \in \mathbb{F}_{\mathrm{cc}}^{(p)}\left(\mathbb{R}^{n}\right)$ for all $i=1,2, \cdots, M$. Then, it follows

$$
\rho_{p}\left(\frac{1}{N} \sum_{i=1}^{N} \widetilde{U}^{i}, \mathcal{E}_{u_{\mathrm{o}}}\left[\widetilde{U}\left(u_{\mathrm{o}}, \cdot\right)\right]\right) \rightarrow 0 \quad \text { a.s. }
$$

as $N \rightarrow \infty$. Hence, the following proposition is obtained $[8,10]$.

[Proposition 4] Let $\left\{\widetilde{U}^{i}\left(u_{\mathrm{o}}, \cdot\right) ; i=1,2, \cdots\right\}$ be a sequence of i.i.d. elementary fuzzy random sets distributed as $\widetilde{U}\left(u_{\mathrm{o}}, \cdot\right)$ with its expectation $\mathcal{E}_{u_{\mathrm{o}}}\left[\widetilde{U}\left(u_{\mathrm{o}}, \cdot\right)\right]$. Then, the SLLN for $\left\{\widetilde{U}^{i}\left(u_{\mathrm{o}}, \cdot\right)\right\}$ is given by eq. (46).
The distribution of an extended fuzzy random set $\widetilde{U}(\omega) \in \widetilde{\mathbb{U}}$ is a probability measure on $\widetilde{\mathbb{U}}$ defined by

$$
P_{\widetilde{U}}(\mathrm{~B})=P\left(\widetilde{U}^{-1}(\mathrm{~B})\right)
$$

for any $\mathrm{B} \in \mathcal{B}$. Let $\mathcal{A}_{\widetilde{U}}$ be the $\sigma$-algebra generated by $\widetilde{U}^{-1}(\mathrm{~B})$, i.e.,

$$
\mathcal{A}_{\widetilde{U}}=\sigma\left\{\widetilde{U}^{-1}(\mathrm{~B}) \in \mathcal{A}_{1} \otimes \mathcal{A}_{2} ; \mathrm{B} \in \mathcal{B}\right\} .
$$

Then, as same as that for the elementary fuzzy random sets, the extended fuzzy random sets $\left\{\widetilde{U}^{i} ; i=\right.$ $1,2, \cdots\}$ are said to be independent if $\left\{\mathcal{A}_{\widetilde{U}^{i}} ; i=1,2, \cdots\right\}$ are independent, and identically distributed if all $\left\{P_{\widetilde{U}^{i}}\right.$; $i=1,2, \cdots\}$ are identical, and independent identically distributed(i.i.d.), if they are independent and identically distributed.

Since $\mathcal{E}[\widetilde{U}] \in \mathbb{F}_{\mathrm{cc}}^{(p)}\left(\mathbb{R}^{n}\right)$, and using eq. (26) it can be rewritten as follows

$$
\mathcal{E}[\tilde{U}]=\sum_{i=0, \pm 1, \pm 2, \cdots} \widetilde{U}_{i} \cdot P\left(\omega_{i}^{(1)}\right) .
$$

Then, substituting $\widetilde{\mathrm{sp}}\left(\widetilde{U}^{i}, \alpha, x\right), \widetilde{\mathrm{sp}}(\widetilde{U}, \alpha, x)$ for $X^{i}$ and $X$ respectively in Proposition 3, we have

$$
\frac{1}{N} \sum_{i=1}^{N} \widetilde{\operatorname{sp}}\left(\widetilde{U}^{i}, \alpha, x\right)=\widetilde{\operatorname{sp}}\left(\frac{1}{N} \sum_{i=1}^{N} \widetilde{U}^{i}, \alpha, x\right)
$$

and

$$
E(\widetilde{\mathrm{sp}}(\widetilde{U}, \alpha, x))=\widetilde{\mathrm{sp}}(\mathcal{E}[\widetilde{U}], \alpha, x) .
$$

Then, if

$$
E\left(\|\widetilde{U}\|_{p}\right)=E\left(\rho_{p}(\widetilde{U},\{0\})\right)<+\infty,
$$

using eq. (49), eq. (50) and eq. (41), we have

$$
\begin{aligned}
& \left\|\widetilde{\operatorname{sp}}\left(\frac{1}{N} \sum_{i=1}^{N} \widetilde{U}^{i}, \alpha, x\right)-\widetilde{\operatorname{sp}}(\mathcal{E}[\widetilde{U}], \alpha, x)\right\|_{p} \\
& =\rho_{p}\left(\frac{1}{N} \sum_{i=1}^{N} \widetilde{U}^{i}, \mathcal{E}[\widetilde{U}]\right) \rightarrow 0 \text { a.s. as } N \rightarrow \infty
\end{aligned}
$$

Then, the following proposition is obtained.

[Proposition 5] Let $\left\{\widetilde{U}^{i} ; i=1,2, \cdots\right\}$ be a sequence of i.i.d. extended fuzzy random sets distributed as $\widetilde{U}$ with its expectation $\mathcal{E}[\widetilde{U}]$. Then, if $E\left(\|\widetilde{U}\|_{p}\right)<+\infty$, the SLLN for $\left\{\widetilde{U}^{i}\right\}$ is given by eq. (52).

\section{Numerical Examples for Vague Perception of Random Phenomena}

Since the simulation results for vague perceptions of crisp phenomena have already been shown in[10], those only for the vague perceptions of random phenomena are shown here, which are revised results given in $[9]$. 


\subsection{Approximation of Set Representa- tion by Step-wise Levels}

The set representation of an extended fuzzy random set as the vague perception of a random phenomenon given by eq. (23) is approximated by the step-wise membership levels, i.e.,

$$
[\widetilde{U}(\omega)]=\left\{[\widetilde{U}(\omega)]_{\alpha_{k}} \mid k=0,1,2, \cdots, L\right\},
$$

where $0=\alpha_{0}<\alpha_{1}<\alpha_{2}<\cdots<\alpha_{L}=1$ and $[\widetilde{U}(\omega)]_{\alpha_{k}}$ is given by

$$
[\widetilde{U}(\omega)]_{\alpha_{k}}=\left\{x \mid\left(x \in \mathbb{R}^{n}\right) \wedge\left((\widetilde{U}(\omega))(x) \geq \alpha_{k}\right)\right\}
$$

for $k=1,2, \cdots, L$, and

$$
\begin{aligned}
{[\widetilde{U}(\omega)]_{\alpha_{0}} } & =[\widetilde{U}(\omega)]_{0} \\
& =\operatorname{cl} .\left\{x \mid\left(x \in \mathbb{R}^{n}\right) \wedge((\widetilde{U}(\omega))(x)>0)\right\}
\end{aligned}
$$

satisfying

$$
\begin{aligned}
{[\widetilde{U}(\omega)]_{1} } & =[\widetilde{U}(\omega)]_{\alpha_{L}} \subseteq[\widetilde{U}(\omega)]_{\alpha_{L-1}} \subseteq \cdots \\
& \subseteq[\widetilde{U}(\omega)]_{\alpha_{1}} \subseteq[\widetilde{U}(\omega)]_{\alpha_{0}}=[\widetilde{U}(\omega)]_{0}
\end{aligned}
$$

Hence, the set representations of $\left\{\widetilde{U}_{j} ; j=0, \pm 1, \pm 2, \cdots\right\}$ in eq. (26) are also approximated by the step-wise membership levels, i.e.,

$$
\left[\widetilde{U}_{j}\right]=\left\{\left[\widetilde{U}_{j}\right]_{\alpha_{k}} \mid k=0,1,2, \cdots, L\right\}
$$

and $\left[\widetilde{U}_{j}\right]_{\alpha_{k}}$ is given by

$$
\left[\widetilde{U}_{j}\right]_{\alpha_{k}}=\left\{x \mid\left(x \in \mathbb{R}^{n}\right) \wedge\left(\left(\widetilde{U}_{j}\right)(x) \geq \alpha_{k}\right)\right\}
$$

for $k=1,2, \cdots, L$, and

$$
\left[\widetilde{U}_{j}\right]_{\alpha_{0}}=\left[\widetilde{U}_{j}\right]_{0}=\operatorname{cl} .\left\{x \mid\left(x \in \mathbb{R}^{n}\right) \wedge\left(\left(\widetilde{U}_{j}\right)(x)>0\right)\right\}
$$

satisfying

$$
\begin{aligned}
{\left[\widetilde{U}_{j}\right]_{1}=\left[\widetilde{U}_{j}\right]_{\alpha_{L}} \subseteq\left[\widetilde{U}_{j}\right]_{\alpha_{L-1}} } & \subseteq \\
\subseteq & \cdots \subseteq\left[\widetilde{U}_{j}\right]_{\alpha_{1}} \subseteq\left[\widetilde{U}_{j}\right]_{\alpha_{0}}=\left[\widetilde{U}_{j}\right]_{0} .
\end{aligned}
$$

Let $\left\{\widetilde{U}^{i}(\cdot) ; i=1,2, \cdots\right\}$ be a sequence of i.i.d. elementary fuzzy random sets distributed as $\widetilde{U}(\cdot)$ with its expectation $\mathcal{E}[\widetilde{U}(\cdot)]$. Then, using the support function and the metric $\rho_{p}$ for fuzzy sets, we have

$$
\begin{aligned}
& \rho_{p}\left(\frac{1}{N} \sum_{i=1}^{N} \widetilde{U}^{i}, \mathcal{E}[\widetilde{U}(\cdot)]\right)= \\
& \left(\sum_{k=1}^{L} \Delta \alpha_{k} \int_{\mathbb{S}^{n-1}} \mid \frac{1}{N} \sum_{i=1}^{N} \operatorname{sp}\left(x,\left[\widetilde{U}^{i}\right]_{\alpha_{k}}\right)\right. \\
& \left.\quad-\left.\operatorname{sp}\left(x, E\left[[\widetilde{U}(\cdot)]_{\alpha_{k}}\right]\right)\right|^{p} d \mu_{\mathbb{S}^{n-1}}(x)\right)^{\frac{1}{p}},
\end{aligned}
$$

where $\Delta \alpha_{k}=\alpha_{k}-\alpha_{k-1}(k=1,2, \cdots, L)$.

\subsection{Simulation Studies}

As stated in Sec. 3, $\left(\Omega_{1}, \mathcal{A}_{1}, P_{1}\right)$ is an elementary probability space describing the randomness of capricious persons' minds, and $\left(\Omega_{2}, \mathcal{A}_{2}, P_{2}\right)$ is a probability space, on which the original random point $u_{\mathrm{o}} \in \mathbb{R}^{n}$ as the model of a random phenomenon is defined. Then, the extended fuzzy random set as a vague perception of the original random point $u_{\mathrm{o}}$ is defined on $(\Omega, \mathcal{A}, P)=\left(\Omega_{1} \times \Omega_{2}, \mathcal{A}_{1} \otimes \mathcal{A}_{2}, P_{1} \times P_{2}\right)$ and given by eq. (23).

In simulation studies, the original random point $u_{\mathrm{o}}\left(\omega^{(2)}\right) \in \mathbb{R}$ is assumed to be a Gaussian random variable(i.e., $n=1$ ) with mean $m_{u}$, the variance $\sigma_{u}^{2}$, and its realization is recognized to be in some interval $I_{j}(j=0, \pm 1, \pm 2, \cdots)$ such that

$$
I_{j}=\left[\frac{2 j-1}{4} \sigma_{u}, \frac{2 j+1}{4} \sigma_{u}\right) .
$$

The subset $A_{j}$ is given by

$$
A_{j}=\Omega_{1} \times A_{j}^{(2)}
$$

and

$$
A_{j}^{(2)}=\left\{\omega^{(2)} \in \Omega_{2} \mid u_{\mathrm{o}}\left(\omega^{(2)}\right) \in I_{j}\right\} .
$$

Then, the sub $\sigma$-algebra $\mathcal{S} \subset \mathcal{A}_{1} \otimes \mathcal{A}_{2}$ given in Sec. 3 is generated by the cylinder sets of the form $\left\{A_{j} ; j=\right.$ $0, \pm 1, \pm 2, \cdots\}$. The conditional probability $P(B \mid \mathcal{S})$ is given by

$$
P(B \mid \mathcal{S})=\sum_{j=0, \pm 1, \pm 2, \cdots} \mathbf{1}_{A_{j}}(\omega) \cdot \frac{P\left(B \cap A_{j}\right)}{P\left(A_{j}\right)}
$$

for $\forall B \in \mathcal{A}$. Hence, if $\omega \in A_{j}$, it follows

$$
P(B \mid \mathcal{S})=P\left(B \mid A_{j}\right)=\frac{P\left(B \cap A_{j}\right)}{P\left(A_{j}\right)} .
$$

Let $B_{i}$ be given by

$$
\Omega=\bigcup_{i} B_{i} \quad \text { and } \quad B_{i}=\left\{\omega_{i}^{(1)}\right\} \times \Omega_{2} .
$$

Then, for $\omega \in A_{j}$, we have

$$
\begin{aligned}
\mathcal{E}[\widetilde{U} \mid \mathcal{S}](\omega) & =\mathcal{E}\left[\sum_{i} \widetilde{U}_{i} \cdot \mathbf{1}_{\omega_{i}^{(1)}}(\omega) \mid \mathcal{S}\right](\omega) \\
& =\sum_{i=0, \pm 1 \pm 2, \cdots} \widetilde{U}_{i} \cdot P\left(B_{i} \mid A_{j}\right) .
\end{aligned}
$$

The set representation of $\widetilde{U}_{i}$ is assumed to be given by

$$
\begin{aligned}
{\left[\widetilde{U}_{i}\right]_{\alpha_{k}}=\left[a_{i}-\frac{\ell_{i}}{\pi} \cos ^{-1}\left(2 \alpha_{k}-1\right)\right.} & \\
& \left.a_{i}+\frac{r_{i}}{\pi} \cos ^{-1}\left(2 \alpha_{k}-1\right)\right] .
\end{aligned}
$$

The reconstructed membership function of $\widetilde{U}_{i}$ from eq. (67) is given by 


$$
\left(\widetilde{U}_{i}\right)(x)=\left\{\begin{array}{c}
\frac{1}{2}\left\{\begin{array}{c}
\left.\cos \left(-\frac{\pi}{\ell_{i}}\left(x-a_{i}\right)+1\right)\right\} \\
\text { for } a_{i}-\ell_{i} \leq x \leq a_{i}
\end{array}\right. \\
\frac{1}{2}\left\{\begin{array}{c}
\left.\cos \left(\frac{\pi}{r_{i}}\left(x-a_{i}\right)+1\right)\right\} \\
\text { for } a_{i} \leq x \leq a_{i}+r_{i}
\end{array}\right. \\
0 \quad \text { otherwise },
\end{array}\right.
$$

and its shape is illustrated in Fig. 1.

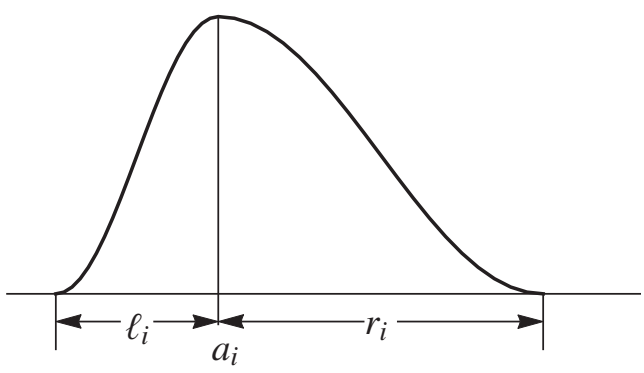

Fig. 1 Membership function of $\left(\widetilde{U}_{i}\right)(x)$

In order to describe the capricious mind of each individual, which may fluctuates slightly but randomly, the conditional probabilities $P\left(B_{i} \mid A_{j}\right) i=0, \pm 1, \pm 2, \cdots$ in eq. (66) are assumed to be given by

$$
\begin{array}{ll}
P\left(B_{j-2} \mid A_{j}\right)=0.2, & P\left(B_{j-1} \mid A_{j}\right)=0.2, \\
P\left(B_{j} \mid A_{j}\right)=0.3, & P\left(B_{j+1} \mid A_{j}\right)=0.2, \\
P\left(B_{j+2} \mid A_{j}\right)=0.1 &
\end{array}
$$

at each $j$, and otherwise, $P\left(B_{i} \mid A_{j}\right)=0$. The number of data $N$ is set as $N=100$, the number of levels $L$ is set as $L=50$, and $\Delta \alpha_{k}$ in eq. (59) is given by $\Delta \alpha_{k}=1 / L=0.02$. Furthermore, the mean $m_{u}$ and the variance $\sigma_{u}^{2}$ of the original random point $u_{\mathrm{o}}$ are set as $m_{u}=0$ and $\sigma_{u}^{2}=10$, and the set of parameters $\left(a_{i}, \ell_{i}, r_{i}\right)$ in eq. (67) are assumed to be given by

$$
a_{i}=\frac{i}{4} \sigma_{u}, \quad \ell_{i}=\frac{3}{4} \sigma_{u}, \quad r_{i}=\frac{3}{4} \sigma_{u} .
$$

Then, using eq. (66) and eq. (69), the conditional expectation of $\widetilde{U}$ at $\omega \in A_{j}$ is given by

$$
\begin{aligned}
\mathcal{E}[\widetilde{U} \mid \mathcal{S}](\omega)=0.2 \widetilde{U}_{j-2}+0.2 \widetilde{U}_{j-1}+0.3 \widetilde{U}_{j} \\
+0.2 \widetilde{U}_{j+1}+0.1 \widetilde{U}_{j+2}
\end{aligned}
$$

with its set representation

$$
[\mathcal{E}[\widetilde{U} \mid \mathcal{S}](\omega)]=\left\{[\mathcal{E}[\widetilde{U} \mid \mathcal{S}](\omega)]_{\alpha_{k}} \mid k=1,2, \cdots, L\right\}
$$

and

$$
\begin{aligned}
& {[\mathcal{E}[\widetilde{U} \mid \mathcal{S}](\omega)]_{\alpha_{k}}=} E\left[[\widetilde{U}]_{\alpha_{k}} \mid \mathcal{S}\right](\omega) \\
&=0.2\left[\widetilde{U}_{j-2}\right]_{\alpha_{k}}+0.2\left[\widetilde{U}_{j-1}\right]_{\alpha_{k}}+0.3\left[\widetilde{U}_{j}\right]_{\alpha_{k}} \\
&+0.2\left[\widetilde{U}_{j+1}\right]_{\alpha_{k}}+0.1\left[\widetilde{U}_{j+2}\right]_{\alpha_{k}} .
\end{aligned}
$$

Then, the expectation of $\widetilde{U}$ is given by

$$
\mathcal{E}[\widetilde{U}]=\mathcal{E}[\mathcal{E}[\widetilde{U} \mid \mathcal{S}]]=\sum_{j=0, \pm 1 \pm 2, \cdots} \mathcal{E}[\widetilde{U} \mid \mathcal{S}](\omega) P\left(A_{j}\right)
$$

with

$$
P\left(A_{j}\right)=\frac{1}{\sqrt{2 \pi} \sigma_{u}} \int_{I_{j}} \exp \left\{-\frac{\left(x-m_{u}\right)^{2}}{2 \sigma_{u}^{2}}\right\} d x .
$$

The shapes of the membership functions of $\widetilde{U}_{i}(i=$ $0, \pm 1, \pm 2, \cdots)$ and $\mathcal{E}[\widetilde{U}]$ are given in Fig. 2.

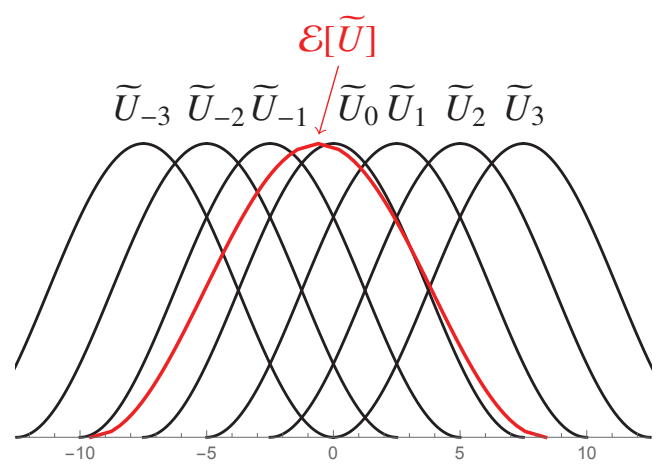

Fig. 2 Shapes of $\left(\widetilde{U}_{i}\right)(x)(i=0, \pm 1, \pm 2, \cdots)$ and $(\mathcal{E}[\widetilde{U}])(x)$

The membership functions of the estimated expectations of $\mathcal{E}[\widetilde{U}]$ are depicted in Fig. 3, where the black lines denote the estimated ones at every 2 steps whereas the red lines denote the true one depicted only for the convenience of the comparisons between the estimated and true ones.

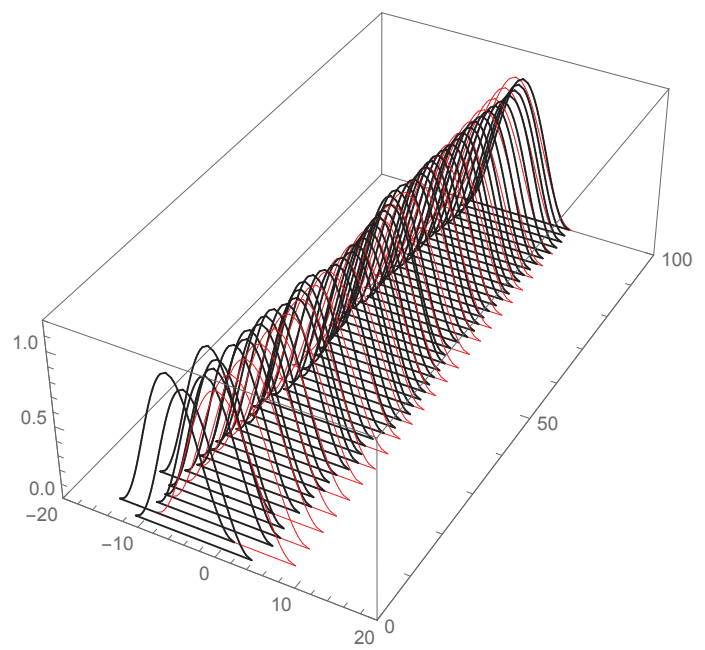

Fig. 3 Membership functions of estimated expectation of $\mathcal{E}[\widetilde{U}]$

Since the support function of the closed interval $[a, b]$ is given by

$$
\operatorname{sp}(x,[a, b])= \begin{cases}-a & \text { if } x=-1 \\ b & \text { if } x=1\end{cases}
$$

those of $\left\{\widetilde{U}_{i}\right\}$ are given by

$$
\widetilde{\mathrm{sp}}\left(\widetilde{U}_{i}, \alpha_{k}, x\right)= \begin{cases}\operatorname{sp}\left(x,\left[\widetilde{U}_{i}\right]_{\alpha_{k}}\right) & \text { for } k=1,2, \cdots, L \\ 0 & \text { for } k=0,\end{cases}
$$




$$
\operatorname{sp}\left(x,\left[\widetilde{U}_{i}\right]_{\alpha_{k}}\right)=\left\{\begin{array}{c}
-\left(a_{i}-\frac{\ell_{i}}{\pi} \cos ^{-1}\left(2 \alpha_{k}-1\right)\right) \\
\text { for } x=-1 \\
a_{i}+\frac{r_{i}}{\pi} \cos ^{-1}\left(2 \alpha_{k}-1\right) \\
\text { for } x=1 .
\end{array}\right.
$$

Furthermore, the estimation error eq. (52) with $p=1$ can be rewritten by

$$
\begin{aligned}
& \rho_{1}\left(\frac{1}{N} \sum_{i=1}^{N} \widetilde{U}^{i}, \mathcal{E}[\widetilde{U}]\right)=\frac{1}{L \times 2} \sum_{k=1}^{L} \\
& {\left[\left|\frac{1}{N} \sum_{i=1}^{N} \operatorname{sp}\left(-1,\left[\widetilde{U}^{i}\right]_{\alpha_{k}}\right)-\operatorname{sp}\left(-1, E\left[[\widetilde{U}]_{\alpha_{k}}\right]\right)\right|\right.} \\
& \left.\quad+\left|\frac{1}{N} \sum_{i=1}^{N} \operatorname{sp}\left(1,\left[\widetilde{U}^{i}\right]_{\alpha_{k}}\right)-\operatorname{sp}\left(1, E\left[[\widetilde{U}]_{\alpha_{k}}\right]\right)\right|\right] .
\end{aligned}
$$

Then, using eq. (71) and eq. (74) eq. (78), the estimation error $\rho_{1}$ between the estimated expectation of the fuzzy random set $\widetilde{U}$ and true one is calculated. The simulation result up to $N=100$ is depicted in Fig. 4.

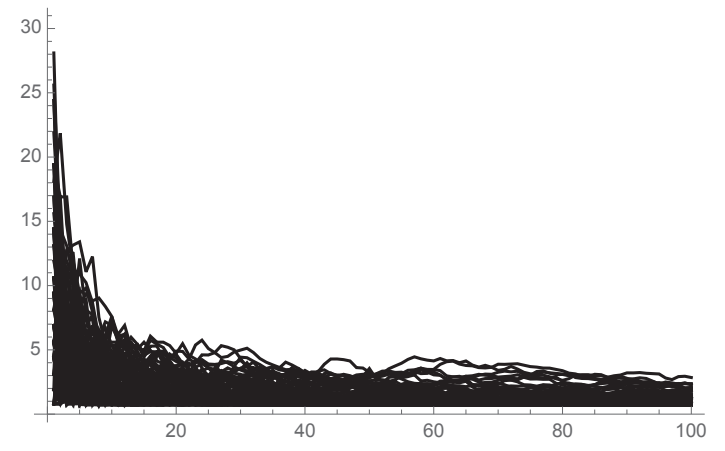

Fig. 4 Estimation errors $\rho_{1}$ (100 sample paths)

\section{Conclusions}

In this paper, the author has investigated the convergent properties of estimators concerned with a class of vague random phenomena, where the fuzzy random set is considered as a model of the capricious vague perception of a crisp phenomenon or a crisp random phenomenon.

First, fuzzy random sets as vague random perceptions of non-random or random phenomena have been investigated, and their expectations have been also considered. Secondly applying the standard Strong Law of Large Numbers(SLLN) for the random elements in a separable Banach space, the convergent properties of estimators for expectations of the proposed fuzzy random sets have been theoretically examined, and finally they have been confirmed numerically by simulation studies.
The proposed fuzzy random sets are expected to apply to the social system modelings, where many data have both the properties of vagueness and randomness.

\section{References}

[1] H. Kwakernaak: Fuzzy random variables-I: Definitions and theorems; Information Sciences, Vol. 15, pp. 1-29 (1978)

[2] R. Kruse, E. Schwecke and J. Heinsohn: Uncertainty and Vagueness in Knowledge Based Systems, John Wiley \& Sons (1994)

[3] M. L. Puri and D. A. Ralescu: Fuzzy random variables; Journal of Mathematical Analysis and its Applications, Vol. 114, pp. 409-422 (1986)

[4] Y. Ogura, S. Li and S. V. Kreinovich: Limit Theorems and Applications of Set-Valued and Fuzzy SetValued Random Variables, Kluwer Academic Publishers (2002)

[5] V. Krätschmer: Integrals of random fuzzy sets; Sociedad de Estaística e Investigación Operativa Test, Vol. 15, No. 2, pp. 433-469 (2006)

[6] T. Fukuda: Probabilistic approach for vague perceptions of complex phenomena; Proceedings of The $43 \mathrm{rd}$ ISCIE International Symposium on Stochastic Systems Theory and its Applications, pp. 101-106 (2012)

[7] T. Fukuda: On a probabilistic approach for capricious vague perceptions of random phenomena; Transactions of ISCIE, Vol. 25, No. 12, pp. 382-388 (2012)

[8] T. Fukuda: On convergences of estimates concerned with fuzzy random data; Proceedings of The 45th ISCIE International Symposium on Stochastic Systems Theory and its Applications, pp. 361-366 (2014)

[9] T. Fukuda: Simulation studies for a class of fuzzy random data; Proceedings of The 46th ISCIE International Symposium on Stochastic Systems Theory and its Applications, pp. 118-123 (2015)

[10] T. Fukuda: On estimation problem associated with persons' capricious perceptions of crisp phenomena; Otemon Economic Studies, Vol. 46, pp. 1-36 (2013)

[11] M. Ledoux and M. Talagrand: Probability in Banach Spaces, Springer (2002)

\section{Author}

\section{Tokuo Fukuda (Member)}

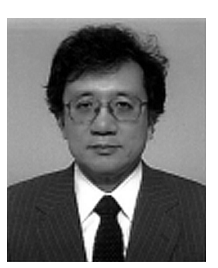

Tokuo FUKUDA received the B.S. and M.S. degree in engineering from Kyoto Institute of Technology, Kyoto, Japan in 1975 and 1977 respectively and he received the Ph.D. degree in engineering from Osaka University, Osaka, Japan in 1987. He is now a Professor at Otemon Gakuin University, Osaka, Japan. He is a member of IEEE, ISCIE, SICE, SOFT and Japan SIAM. 